\title{
Incipient Diabetes Treated with Long-Term Classical Prescription
}

\author{
Xiuxiu Wei $\mathbb{D},{ }^{1,2}$ Jiaxing Tian $\mathbb{D},{ }^{1}$ Xinmiao Wang, ${ }^{1}$ Haoran $W u\left(\mathbb{D},{ }^{1,2}\right.$ Haiyu Zhang, ${ }^{1,2}$ \\ and Xiaolin Tong $\mathbb{B D}^{1}$ \\ ${ }^{1}$ Department of Endocrinology, Guang'anmen Hospital, China Academy of Chinese Medical Sciences, Beijing 100053, China \\ ${ }^{2}$ Graduate College, Beijing University of Traditional Chinese Medicine, Beijing 100029, China
}

Correspondence should be addressed to Jiaxing Tian; tina_yai@163.com and Xiaolin Tong; tongxiaolin@vip.163.com

Received 10 July 2019; Revised 16 September 2019; Accepted 9 October 2019; Published 5 November 2019

Guest Editor: Ruozhi Zhao

Copyright (c) 2019 Xiuxiu Wei et al. This is an open access article distributed under the Creative Commons Attribution License, which permits unrestricted use, distribution, and reproduction in any medium, provided the original work is properly cited.

\begin{abstract}
Background. Diabetes mellitus (DM) belongs to the progressive and irreversible disease. With the development of the disease, the function of beta cells declines significantly. Current treatments cannot reverse the course of the disease. The role of traditional Chinese medicine (TCM) in the DM treatment has been recognized widely, while there are few long-term observation reports. In this study, we introduced a case of DM treated by classical prescription alone for 10 years, which would provide the reference for clinical practice. Case Presentation. A 64-year-old male complained of a 2-month history of dry mouth, frequent urination, and blurred vision and found increased blood glucose for 3 days. The patient's glycated hemoglobin was $14.2 \%$, fasting plasma glucose fluctuated at $12-15 \mathrm{mmol} / \mathrm{L}$, and urinary albumin excretion rate (UAER) was $32.9 \mu \mathrm{g} / \mathrm{min}$. The male was treated with 10 years of continuous classical prescription alone. After 3 months of TCM treatment, the patient's blood glucose level decreased significantly and blurred vision symptoms improved. With continued TCM treatment, his UAER normalized. Subsequently, he continued outpatient consultation, and his TCM prescription was adjusted according to clinical symptoms. After 10 years of continuous TCM treatment, his blood glucose remained stable, urinary microalbumin quantitation showed no abnormalities, and blurred vision disappeared. Conclusions. This case provides specific treatment plans and effective references for long-term control of blood glucose, prevention and treatment of diabetes complications, delay of disease progression, and protection of impaired islet function in the treatment of diabetes with TCM. TCM may become a meaningful alternative DM treatment in the future.
\end{abstract}

\section{Background}

According to the International Diabetes Federation in 2017, nearly $9.9 \%$ of the world's population or 693 million people will be suffering from diabetes mellitus (DM) by 2045 [1]. With the aging of the population and changes in lifestyle in China, diabetes has reached a prevalence of $10.9 \%$, accounting for one-third of the total number of diabetic patients in the world [2]. DM can cause severe microvascular and macrovascular complications. Within 10 years after the diagnosis of diabetes, diabetic neuropathy is found in $60 \%$ $90 \%$ of cases [3], and $40 \%$ of end-stage renal disease cases are due to diabetes in the United States [4]. Furthermore, more than $60 \%$ of patients with diabetes develop retinopathy within 10 years, approaching nearly $100 \%$ by 15 years [5]. Given the chronic and severe complications of diabetes, the disease carries a heavy social, financial, and healthcare systems burden. With a prolonged course, pancreatic beta cells are gradually destroyed, insulin synthesis and secretion are permanently reduced, and islet dysfunction gradually becomes irreversible [6].

However, the clinical treatment and control of T2DM and its complications have unsatisfactory side effects. Glyburide causes hypoglycemia significantly more frequently than other oral medications [7]. Sulfonylureas, particularly glimepiride, have the highest proportion of adverse reactions (up to $32.5 \%$ ), mainly severe hypoglycemia. The next highest proportion of adverse reactions are reported for acarbose (17.2\%), mainly gastrointestinal reactions. Pioglitazone (11.3\%) mainly causes damage to the cardiovascular system. Other adverse drug reactions include lactic acidosis caused by biguanides, edema, and weight gain caused by 
thiazolidinediones, such as rosiglitazone [8]. It has been reported that although patients taking empagliflozin/linagliptin show significant hypoglycemic effects without developing severe hypoglycemia, they carry a high risk of urinary tract and genital infections [9]. Therefore, it is important to explore safer and effective treatment methods.

There is a long history in treating DM by traditional Chinese medicine (TCM). Serious of large-scale clinical randomized controlled trials (RCTs) have demonstrated the efficacy of TCM in antidiabetes and improving islet function. Dachaihu decoction and Gegen Qinlian decoction, as classical prescriptions, have shown that they are effective in controlling blood glucose level and are safe to use in DM patients by RCTs [10, 11]. A retrospective study of 142 diabetic patients for at least 6 months proved that classical prescription could reduce the use of antidiabetes drugs. In addition, Chinese herbal decoction significantly enhanced the hypoglycemic action and had a certain effect on protecting islet cell function [12]. A 12-week randomized double-blind controlled trial of 186 patients with diabetes mellitus showed that the Chinese herbal medicine Jinlida significantly improved hypoglycemic action compared with placebo on the basis of metformin. Furthermore, Jinlida showed improved $\beta$-cell function with a HOMA- $\beta$ increase $[13,14]$. The study included 800 patients with unsatisfactory glycemic control; treatment with Xiaoke Pill led to significant reduction in the risk of hypoglycemia and similar improvements in glycemic control compared to glibenclamide [15]. However, we did not have a long enough observation course in clinical trials.

This paper describes a case of primary T2DM treated with classical prescription alone for 10 years; the treatment significantly reduced blood glucose, normalized urinary protein levels, improved blurred vision, controlled the occurrence of vascular complications, protected damaged islet function, and delayed the course of diabetes. Blood glucose levels were well controlled after a long-term follow-up treatment. Once the patient's condition was stabilized, the form of TCM powder, which is suitable for long-term use, was adopted as it was easy to prepare, which enhanced compliance and achieved good clinical efficacy.

\section{Case Presentation}

A male patient (age: 64 years, height: $171 \mathrm{~cm}$, weight: $72 \mathrm{~kg}$, body mass index: $24.6 \mathrm{~kg} / \mathrm{m}^{2}$ ) first visited our endocrinology clinic in November 2007 after having been diagnosed with T2DM 3 days earlier at another hospital. At that visit, his glycated hemoglobin (HbAlc) was $14.2 \%$, fasting plasma glucose (FPG) was $14.7 \mathrm{mmol} / \mathrm{L}$, and urinary albumin excretion rate (UAER) was $32.9 \mu \mathrm{g} / \mathrm{min}$. Routine urine analysis showed GLU (+) $5.5 \mathrm{mmol} / \mathrm{L}$. A fundus examination yielded negative results. Before diagnosis, he had polydipsia, polyuria, and weight loss. When he visited our clinic, he complained of dry mouth and blurred vision and took oral medication for hypoglycemia for only 1 day (acarbose $50 \mathrm{mg}$ and glimepiride $1 \mathrm{mg}, 3$ times a day).

We advised the patient to stop taking oral hypoglycemic drugs and change to TCM treatment. We prescribed a formula consisting of Chaihu (Radix Bupleuri) $15 \mathrm{~g}$, Huangqin (Radix Scutellariae Baicalensis) $30 \mathrm{~g}$, Qingbanxia (Pinelliae Rhizoma Praeparatum Cum Alumine) $15 \mathrm{~g}$, Zhishi (Fructus Aurantii Immaturus) $15 \mathrm{~g}$, Dahuang (Radix Et Rhizoma Rhei Palmati) $3 \mathrm{~g}$, Huanglian (Rhizoma Coptidis) $45 \mathrm{~g}$, Ganjiang (Rhizoma Zingiberis Recens) 9g, and Zhimu (Common Anemarrhena Rhizome) $30 \mathrm{~g}$.

The patient returned for follow-up after 1 month of treatment. His HbAlc was $11.0 \%$ and UAER was $31.53 \mu \mathrm{g} / \mathrm{min}$. During the first 2 weeks of the medication, his FPG fluctuated between 6.7 and $9.2 \mathrm{mmol} / \mathrm{L}$ and his $2 \mathrm{~h}$ plasma glucose (2hPG) fluctuated between 5.1 and $13.3 \mathrm{mmol} / \mathrm{L}$. In the subsequent 2 weeks, his FPG fluctuated between 6.0 and $7.4 \mathrm{mmol} / \mathrm{L}$ and $2 \mathrm{hPG}$ fluctuated between 4.9 and $9.6 \mathrm{mmol} / \mathrm{L}$. His blurred vision was relieved. As the patient still reported symptoms of dry mouth and early awakening, we added Suanzaoren (Ziziphi Spinosae Semen) $30 \mathrm{~g}$, Shuizhi (Hirudo) $9 \mathrm{~g}$, and Chishao (Radix Paeoniae Rubra) $30 \mathrm{~g}$ to the original preparation.

In January 2008, the patient returned for follow-up after another month of the modified treatment. His HbAlc was $7.1 \%$, FPG was $6.4 \mathrm{mmol} / \mathrm{L}, 2 \mathrm{hPG}$ was $7.1 \mathrm{mmol} / \mathrm{L}$, and UAER was $17.11 \mu \mathrm{g} / \mathrm{min}$ (within the normal range). Moreover, his sleep symptoms improved.

In February 2008, the patient visited our hospital for the fourth time. His FPG was $5.3 \mathrm{mmol} / \mathrm{L}, 2 \mathrm{hPG}$ was $7.7 \mathrm{mmol} / \mathrm{L}$, and bedtime blood glucose was $8.2 \mathrm{mmol} / \mathrm{L}$. The patient's blurred vision had disappeared, and he had no other particular discomfort. We adjusted the prescription to Zhimu (Common Anemarrhena Rhizome) $30 \mathrm{~g}$, Huangqin (Radix Scutellariae Baicalensis) $15 \mathrm{~g}$, Huanglian (Rhizoma Coptidis) $30 \mathrm{~g}$, Rougui (Cinnamomum cassia Presl) $15 \mathrm{~g}$, Shengdihuang (Rehmanniae Radix) 60g, Shuizhi (Hirudo) 9g, Suanzaoren (Ziziphi Spinosae Semen) $30 \mathrm{~g}$, and Wuweizi (Fructus Schisandra Chinensis) $9 \mathrm{~g}$. The prescription was powdered and prescribed to be taken twice a day, $9 \mathrm{~g}$ each time.

From March 2008 to February 2011, the patient was seen at follow-up visits 9 times. Additional herbal medicine was added or subtracted on the basis of the fourth prescription. The patient's blood glucose was stably controlled, and HbA1c fluctuated between $4.9 \%$ and $6.2 \%$. On March 7, 2011, the patient visited the hospital for the $16^{\text {th }}$ time; his $\mathrm{HbAlc}$ was $5.6 \%$, FPG was $6.1 \mathrm{mmol} / \mathrm{L}$, and $2 \mathrm{hPG}$ was $7.9 \mathrm{mmol} / \mathrm{L}$. The patient continued to use the powdered prescription orally.

From April 2011 to January 2015, the patient was followed up another 5 times, during which further modifications were made to the prescription at the $16^{\text {th }}$ visit. The patient's blood glucose control remained stable, and the $\mathrm{HbAlc}$ fluctuated between $5.6 \%$ and $6.5 \%$. His urinary microalbumin/urinary creatinine was $8.31 \mathrm{mg} / \mathrm{g}$. Fundus examination yielded a negative result for retinopathy.

From June 2016 to June 2018, the patient was followed up 3 times. His main symptom was nocturia (once or twice per night). Considering that the patient is an elderly male and given the chronic course of diabetes, we prescribed a formula consisting of Tianma (Rhizoma Gastrodiae) $180 \mathrm{~g}$, Roucongrong (Cistanche deserticola) $540 \mathrm{~g}$, Ganjiang (Rhizoma Zingiberis Recens) $180 \mathrm{~g}$, Huanglian (Rhizoma Coptidis) $540 \mathrm{~g}$, Zhimu (Common Anemarrhena Rhizome) $540 \mathrm{~g}$, Chishao 


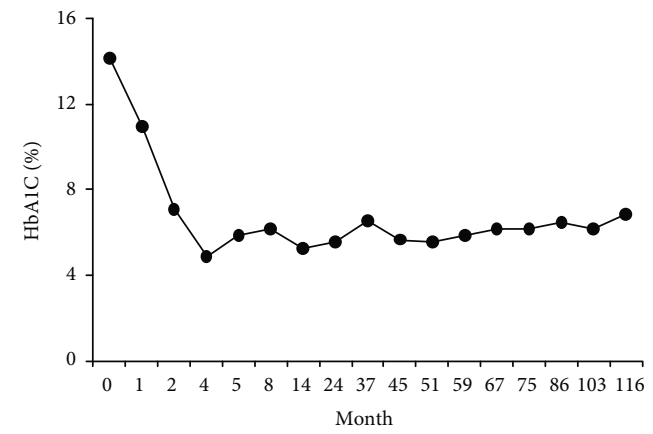

(a)

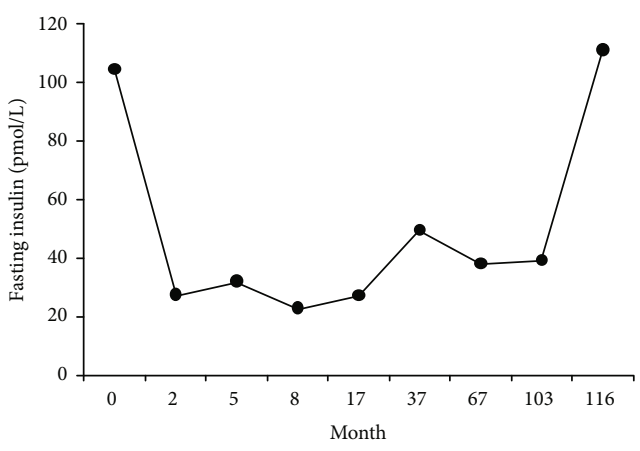

(c)

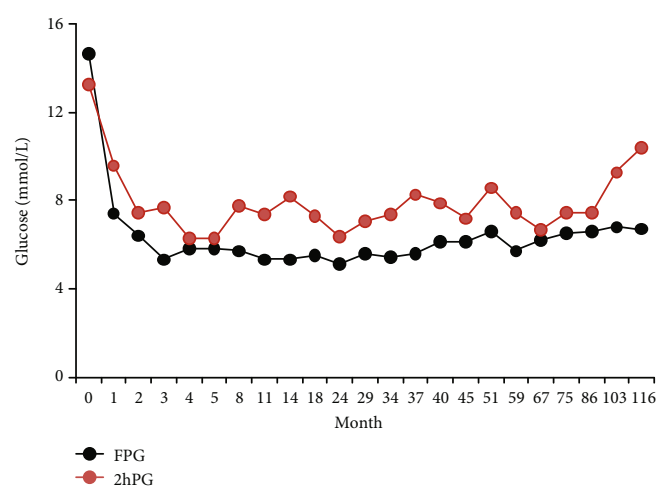

(b)

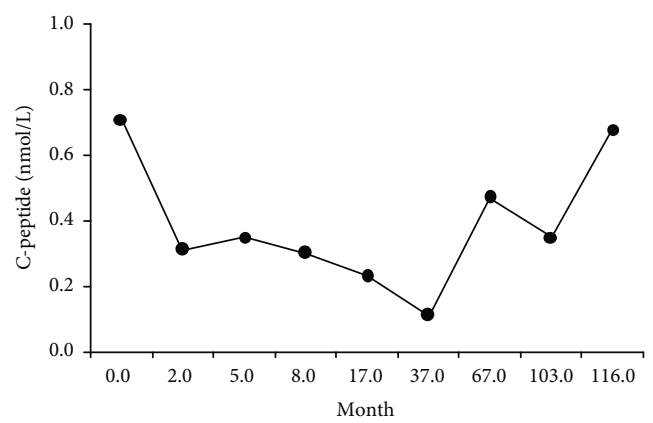

(d)

FIGURE 1: Intervention of TCM decreased blood glucose and improved islet function. (a) Changes in HbAlc: the level of HbAlc decreased significantly after the initial 3 months of treatment and showed a downward trend that tended to be stable after the long-term treatment. (b) Changes in glucose: the level of FPG and 2hPG showed a downward trend and fluctuated within the control standard range. (c) Changes in fasting insulin: the level of fasting insulin improved after the long-term treatment. (d) Changes in C-peptide: the level of Cpeptide improved after the long-term treatment. TCM: traditional Chinese medicine; HbAlc: glycosylated hemoglobin; FPG: fasting plasma glucose; $2 \mathrm{hPG}$ : 2-hour postprandial blood glucose.

(Radix Paeoniae Rubra) $540 \mathrm{~g}$, Shanzhuyu (Fructus Macrocarpii) $540 \mathrm{~g}$, Tianhuafen (Radix Trichosanthis) $540 \mathrm{~g}$, Xiyangshen (Panax quinquefolium) $540 \mathrm{~g}$, and Sanqi (Panax notoginseng) $270 \mathrm{~g}$. The prescription was powdered and prescribed to be taken twice a day ( $9 \mathrm{~g}$ each time). The blood glucose remained stable over a long period, UAER showed no abnormalities, and blurred vision had not returned.

The patient was reviewed every 3 months by telephone follow-up. His HbA1c fluctuated in the range of $6.1 \%-6.5 \%$, FPG fluctuated in the range of 6.5-7 mmol/L, 2hPG remained $<10 \mathrm{mmol} / \mathrm{L}$, and urinary microalbumin/urinary creatinine remained normal over the long term.

The patient is now followed up regularly in our clinic. During follow-up, blood glucose changes (Figure 1; Supplementary Table 2) and islet function (Supplementary Table 1) are reviewed. Blood glucose and vascular complications remained stably controlled. During the 10 years since the initial visit, the patient had not used any oral medication or insulin for blood glucose control, and his HbAlc had reached normal levels by using the TCM powder alone long term. The classical prescription is based on the natural evolution of diabetes, "prediabetes, diabetes, advanced diabetes, and diabetic complications." According to the changes in the patients' clinical symptoms and the characteristics of the chronic disease course, drugs should be adjusted to select the appropriate
TCM with hypoglycemic effects. The HbAlc and blood glucose levels normalized within a short period. We added the herbal medicine to improve circulation during the early stage of treatment to reverse diabetic vascular complications, return urinary microalbumin levels to normal, and improve blurred vision. The patient took TCM to improve hypoglycemia and circulation for a long time, without any adverse reactions or new complications. Medical history and reports in this study had been approved by the patient.

\section{Discussion and Conclusion}

The patient presented here had high blood glucose levels when he was diagnosed with DM (HbA1c, 14.2\%); he also had microalbuminuria and blurred vision caused by elevated blood glucose levels. The patient visited our clinic after receiving oral hypoglycemic drugs for 1 day only, after which he was asked to switch to TCM alone. The patient's blood glucose levels were significantly reduced, microalbuminuria turned to normal, and blurred vision was improved with classical prescription. Although there were signs of clinical proteinuria with renal function injury at the initial diagnosis, the urine protein normalized rapidly after taking the oral decoction and remained normal during follow-up. The TCM was then prepared as a powder, and the patient took 
this with good compliance. During the follow-up treatment with TCM alone for 10 years, his blood glucose control was stable, the urine microalbumin test showed no abnormalities, and no fundus lesions appeared. The patient did not use western medicine, and no adverse reactions such as hypoglycemia occurred. Such cases have rarely been reported. Therefore, this report may provide a reference for the treatment of T2DM in lowering blood glucose and preventing and treating complications with TCM alone.

$\mathrm{T} 2 \mathrm{DM}$ is a progressive metabolic disorder characterized by elevated blood glucose and is characterized by insulin resistance to glucose metabolism, with multiorgan involvement. According to previous studies, the pathophysiological process of diabetes included lipid metabolism disorder, decreased effect of insulin-stimulating hormone, increased basal glucagon level, renal dysregulation, and neurotransmitter dysfunction [16]. Based on the physiological and pathological process of T2DM, western medical treatments range from hypoglycemic drugs, such as sulfonylureas, biguanides, and insulin, to newly developed dipeptidyl peptidase IV inhibitors, glucagon-like peptide-1 receptor agonist, sodium-glucose cotransporter-2 inhibitor, and various insulin analogs. Bariatric surgery and other interventional treatments affect afferent vagus nerve activity- (entero-brain axis-) related control of internal organs, such as the liver and pancreas, to provide obesity-related metabolic control of T2DM [17]. However, long-term oral hypoglycemic drugs or insulin are resistant, and newly developed drugs aggravate the economic burden of patients and easily lead to poor patient compliance. The mechanism of obesity-related T2DM surgery remains unclear, has major limitations, and has not been widely performed. The use of many oral hypoglycemic drugs is limited due to the occurrence of diabetic nephropathy, and insulin therapy also increases body weight, which indirectly increases the risk of diabetic complications. Although these treatments can control blood glucose within the target range, it cannot reverse the process of islet cell failure, and the effects on the associated complications are not obvious.

Studies have suggested that TCM treatment for T2DM is effective, particularly in terms of prevention and reversal of multimetabolic disorders, such as obesity, blood lipids, blood pressure, and fatty liver caused by diabetes $[10,18,19]$. This case reported here had a significantly elevated HbAlc level and diabetic nephropathy urinary protein level, which returned to normal within a short time after the initial 3 months of treatment with TCM only. Once HbA1c was controlled within the target range, the prescription was changed to TCM powder. HbAlc continued a downward trend and stabilized at normal levels with long-term treatment. Furthermore, TCM improved the symptoms of blurred vision, control the occurrence of vascular complications, improved the impaired islet function, and delayed the course of DM.

The ancient Chinese medicine book Huangdi Neijing has recorded the diabetes, while diabetes is thought to be caused by obesity nowadays to a great extent and is divided into four stages: prestage, diabetes stage, middle-late stage, and complication stage. The appropriate TCM should be chosen based on the characteristics of different stages. In a large- scale clinical trial, we have previously found that the classical prescription, Gegen Qinlian decoction, has an independent hypoglycemic effect. Compared with placebo, this decoction reduced HbAlc significantly, and the hypoglycemic effect was positively correlated with the dose used. Gegen Qilian decoction has been shown to reduce glucose by regulating gut microbiota and improving islet function, and its hypoglycemic effect remained after drug withdrawal [11]. In addition, TCM can reduce the risk of complications by addressing multiple targets while reducing blood glucose. In a randomized controlled trial including T2DM patients with abdominal obesity and dyslipidemia, TCM had equivalent efficacy to metformin in lowering glucose and was superior to metformin in improving lipid levels and waist circumference [19].

Due to the multiple vascular complications of diabetes, it has been proposed that DM should be called "TangLuo disease," given the damage to collaterals caused by elevated blood glucose [20]. TCM treatment of DM emphasizes the early improvement of the circulation and is maintained throughout the treatment. This can effectively prevent the occurrence of diabetic complications. In the early stage of treatment, we used drugs to improve circulation; these included Sanqi (Panax notoginseng) and Shuizhi (Hirudo powder). The extracts of Sanqi (Panax notoginseng) have strong anti-inflammatory and antioxidant effects and protect the vascular endothelium [21, 22]. Shuizhi (Hirudo powder) is an animal-derived medicine, which has anticoagulant effects [23]. Shuizhi (Hirudo powder) combined with Huangqi (Radix Astragali Mongolici) is used to treat diabetic nephropathy, as these herbal medicine can protect kidney function, enhance insulin sensitivity, have antioxidant activity, and improve mitochondrial function [24].

With the increasing incidence of obesity, T2DM may become more prevalent in the future. DM has a significant impact on the quality of life of patients and imposes a heavy burden on the finance and public health care system. The use of TCM for treating DM is increasingly gaining attention, with clinical trials demonstrating that herbal medicine can lower blood glucose and provide additional benefits, such as improving insulin resistance and reducing lipid levels and weight $[13-15,25]$, and pharmacological studies showing that TCM can improve islet function, increase insulin secretion, and enhance the use of glucose in peripheral tissues [26-31]. TCM can also be used to treat diabetic complications through improving blood viscosity, microcirculation, and oxidative stress-related abnormalities [32].

Once our patient's condition was stable and blood glucose levels had normalized, we adjusted the prescription to a powder to allow oral administration. Such form is convenient for patients to carry with them, improving long-term medication compliance. In addition, it can effectively prevent and delay the occurrence and progression of complications [33]. The configuration of TCM (powder, formula granule, or pill) can be adjusted flexibly for longterm use, which is more conducive to patient compliance and long-term treatment.

DM is an important chronic, noncommunicable disease. Its treatment and complications not only place a marked 
economic burden on patients but also affect their quality of life. The high incidence of diabetes requires development of a new therapeutic strategy. Early detection and early treatment should be emphasized, and circulatory drugs should be used to improve the prevention and treatment of early complications.

There have been few reports on the treatment of DM with TCM alone. This case report provides evidence of the effective regulation of DM, and reversal and prevention of complications, by using classical prescription alone, and provides a reference for clinical application of TCM treatment. Further studies are needed to confirm whether long-term treatment of TCM can reduce the risk of diabetes complications as well as the safety of long-term TCM application. Additionally, the hypoglycemic, circulatory, and protection for islet function of TCM should be clarified. Nevertheless, this study indicates that TCM may be an important alternative therapy for the prevention and treatment of $\mathrm{DM}$ and its complications.

\section{Abbreviations \\ DM: Diabetes mellitus \\ FPG: Fasting plasma glucose \\ HbA1c: Glycated hemoglobin \\ 2hPG: $2 \mathrm{~h}$ plasma glucose \\ TCM: Traditional Chinese medicine \\ T2DM: Type 2 diabetes mellitus \\ UAER: Urinary albumin excretion rate.}

\section{Data Availability}

The data that support the findings of this study are stored in Guang'anmen Hospital (Beijing, China) and available from the corresponding authors on reasonable request.

\section{Conflicts of Interest}

The authors declare that they have no competing interests.

\section{Authors' Contributions}

XW1 interpreted the data and drafted the manuscript. JT revised the manuscript. XW2, HW, and HZ participated in the data collection. XT presided the project. All authors read and approved the final manuscript.

\section{Acknowledgments}

The authors thank the patient and his family for their participation. This work was partially supported by the Young Elite Scientists Sponsorship Program by CAST (YESS20170034), the National Natural Science Foundation of China (No. 81904187, 81274000), the Special Scientific Research for Traditional Chinese Medicine of China (201507001-11), and the Outstanding Young Scientific and Technological Talents Program (ZZ13-YQ-026).

\section{Supplementary Materials}

Changes in islet function and blood glucose during treatment. (Supplementary Materials)

\section{References}

[1] N. H. Cho, J. E. Shaw, S. Karuranga et al., "IDF diabetes atlas: global estimates of diabetes prevalence for 2017 and projections for 2045," Diabetes Research and Clinical Practice, vol. 138, pp. 271-281, 2018.

[2] L. M. Wang, P. Gao, M. Zhang et al., "Prevalence and ethnic pattern of diabetes and prediabetes in China in 2013," JAMA, vol. 317, no. 24, pp. 2515-2523, 2017.

[3] Chinese Diabetes Society, "Guidelines for the prevention and control of type 2 diabetes in China," Chinese Journal of Diabetes Mellitus, vol. 10, no. 1, pp. 4-67, 2018.

[4] A. J. Collins, B. Kasiske, C. Herzog et al., "United States Renal Data System 2006 Annual Data Report Abstract," American Journal of Kidney Diseases, vol. 49, pp. A6-A7, 2007.

[5] G. L. Chen, Clinical endocrinology, Shanghai Science and Technology Press, 2011.

[6] K. Srinivasan, B. Viswanad, L. Asrat, C. L. Kaul, and P. Ramarao, "Combination of high-fat diet-fed and low-dose streptozotocin-treated rat: a model for type 2 diabetes and pharmacological screening," Pharmacological Research, vol. 52, no. 4, pp. 313-320, 2005.

[7] Y. Peng, S. H. Chen, X. N. Liu, and Q. Y. Sun, "Efficacy of different antidiabetic drugs based on metformin in the treatment of type 2 diabetes mellitus: a network meta-analysis involving eight eligible randomized-controlled trials," Journal of Cellular Physiology, vol. 234, no. 3, pp. 2795-2806, 2019.

[8] F. Li and Z. G. Liu, "Analysis report of 425 cases of adverse reactions of hypoglycemic drugs," Shanxi Medical Journal, vol. 47, no. 14, pp. 1715-1717, 2018.

[9] R. A. Defronzo, C. Lee, and S. Kohler, "Safety and tolerability of combinations of empagliflozin and linagliptin in patients with type 2 diabetes: pooled data from two randomized controlled trials," Advances in Therapy, vol. 35, no. 7, pp. 1009-1022, 2018.

[10] X. L. Tong, S. T. Wu, F. M. Lian et al., "The safety and effectiveness of TM81, a Chinese herbal medicine, in the treatment of type 2 diabetes: a randomized double-blind placebo-controlled trial," Diabetes, Obesity \& Metabolism, vol. 15, no. 5, pp. 448454, 2013.

[11] J. Xu, F. Lian, L. Zhao et al., "Structural modulation of gut microbiota during alleviation of type 2 diabetes with a Chinese herbal formula," The ISME Journal, vol. 9, no. 3, pp. 552-562, 2015.

[12] J. Tian, F. Lian, X. Yu et al., "The efficacy and safety of Chinese herbal decoction in type 2 diabetes: a 5-year retrospective study [J]," Evidence-based Complementary and Alternative Medicine, vol. 2016, Article ID 5473015, 8 pages, 2016.

[13] J. X. Tian, F. M. Lian, L. B. Yang, and X. Tong, "Evaluation of the Chinese herbal medicine Jinlida in type 2 diabetes patients based on stratification: results of subgroup analysis from a 12week trial," Journal of Diabetes, vol. 10, no. 2, pp. 112-120, 2018.

[14] F. M. Lian, J. Tian, X. Chen et al., "The efficacy and safety of Chinese herbal medicine Jinlida as add-on medication in type 2 diabetes patients ineffectively managed by metformin 
monotherapy: a double-blind, randomized, placebo-controlled, multicenter trial," PLoS One, vol. 10, no. 6, 2015.

[15] L. Ji, X. Tong, H. Wang et al., "Efficacy and safety of traditional Chinese medicine for diabetes: a double-blind, randomised, controlled trial," PLoS One, vol. 8, no. 2, p. e56703, 2013.

[16] A. R. Saltiel and C. R. Kahn, "Insulin signalling and the regulation of glucose and lipid metabolism," Nature, vol. 414, no. 6865, pp. 799-806, 2001.

[17] C. Blasi, "The role of the vagal nucleus tractus solitarius in the therapeutic effects of obesity surgery and other interventional therapies on type 2 diabetes," Obesity Surgery, vol. 26, no. 12, pp. 3045-3057, 2016.

[18] F. M. Lian, G. Li, X. Chen et al., "Chinese herbal medicine Tianqi reduces progression from impaired glucose tolerance to diabetes: a double-blind, randomized, placebo-controlled, multicenter trial," The Journal of Clinical Endocrinology \& Metabolism, vol. 99, no. 2, pp. 648-655, 2014.

[19] X. Yu, L. Xu, Q. Zhou et al., "The Efficacy and Safety of the Chinese Herbal Formula, JTTZ, for the Treatment of Type 2 Diabetes with Obesity and Hyperlipidemia: A Multicenter Randomized, Positive-Controlled, Open-Label Clinical Trial," International Journal of Endocrinology, vol. 2018, Article ID 9519231, 11 pages, 2018.

[20] X. L. Tong, Y. Zhao, G. Z. Bi, L. Chen, H. J. Li, and L. Dong, "Discussion on the application of TCM "treatment of disease" and "luplex disease" theory in the treatment of diabetic microvascular complications," Journal of Traditional Chinese Medicine, vol. 48, no. 06, pp. 485-486, 2007.

[21] X. Zhou, V. Razmovski-Naumovski, D. Chang et al., "Synergistic Effects of Danshen (Salvia Miltiorrhiza Radix et Rhizoma) and Sanqi (Notoginseng Radix et Rhizoma) Combination in Inhibiting Inflammation Mediators in RAW264.7 Cells," BioMed Research International, vol. 2016, Article ID 5758195, 12 pages, 2016.

[22] X. Zhou, V. Razmovski-Naumovski, and K. Chan, "A multivariate analysis on the comparison of raw notoginseng (Sanqi) and its granule products by thin-layer chromatography and ultra-performance liquid chromatography," Chinese Medicine, vol. 10, no. 1, 2015.

[23] L. Xiao, J. Nie, D. Li, and K. Chen, "Peptides from two sanguinovorous leeches analyzed by ultra-performance liquid chromatography coupled with electrospray ionization quadrupole time-of-flight mass spectrometric detector," Pharmacognosy Magazine, vol. 11, no. 41, pp. 32-37, 2015.

[24] R. Q. Chen, C. Liao, Q. Guo, L. Wu, L. Zhang, and X. Wang, "Combined systems pharmacology and fecal metabonomics to study the biomarkers and therapeutic mechanism of type 2 diabetic nephropathy treated withAstragalusand leech," RSC Advances, vol. 8, no. 48, pp. 27448-27463, 2018.

[25] J. X. Tian, D. Jin, Q. Bao et al., "Evidence and potential mechanisms of traditional Chinese medicine for the treatment of type 2 diabetes: A systematic review and meta-analysis," Diabetes, Obesity \& Metabolism, vol. 21, no. 8, pp. 18011816, 2019.

[26] C. R. Cederroth, M. Vinciguerra, A. Gjinovci et al., "Dietary phytoestrogens activate AMP-activated protein kinase with improvement in lipid and glucose metabolism," Diabetes, vol. 57, no. 5, pp. 1176-1185, 2008.

[27] S. P. Li, G. H. Zhang, Q. Zeng et al., "Hypoglycemic activity of polysaccharide, with antioxidation, isolated from cultured Cordyceps mycelia," Phytomedicine, vol. 13, no. 6, pp. 428433, 2006.
[28] C. H. Jung, H. M. Seog, I. W. Choi, H. D. Choi, and H. Y. Cho, "Effects of wild ginseng (Panax ginseng C.A. Meyer) leaves on lipid peroxidation levels and antioxidant enzyme activities in streptozotocin diabetic rats," Journal of Ethnopharmacology, vol. 98, no. 3, pp. 245-250, 2005.

[29] M. P. Lu, R. Wang, X. Song, X. Wang, L. Wu, and Q. H. Meng, "Modulation of methylglyoxal and glutathione by soybean isoflavones in mild streptozotocin-induced diabetic rats," Nutrition, Metabolism and Cardiovascular Diseases, vol. 18, no. 9, pp. 618-623, 2008.

[30] H. S. Zhen, J. Liang, and F. Zhou, "Studies on antihyperglycemic effect of ethanolic extracts from Gymnema sylvestre(Retz.)Schult," Chinese Journal of Experimental Traditional Medical Formulae, vol. 13, no. 01, pp. 32-34, 2007.

[31] H. Cao, M. M. Polansky, and R. A. Anderson, "Cinnamon extract and polyphenols affect the expression of tristetraprolin, insulin receptor, and glucose transporter 4 in mouse 3T3-L1 adipocytes," Archives of Biochemistry and Biophysics, vol. 459, no. 2, pp. 214-222, 2007.

[32] T. F. Tzeng, S. S. Liou, and I. M. Liu, "The selected traditional Chinese medicinal formulas for treating diabetic nephropathy: perspective of modern science," Journal of Traditional and Complementary Medicine, vol. 3, no. 3, pp. 152-158, 2013.

[33] X. L. Tong, Z. P. Peng, Y. Z. Jiao, J. C. Zhang, F. Liu, and Q. Zhou, "General situation and review of research on "San" of traditional Chinese medicine," Journal of Traditional Chinese Medicine, vol. 54, no. 01, pp. 12-16, 2013. 


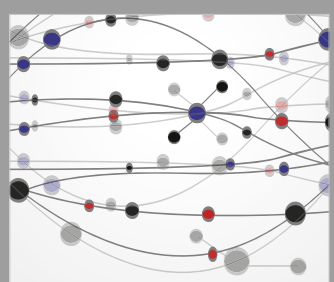

The Scientific World Journal
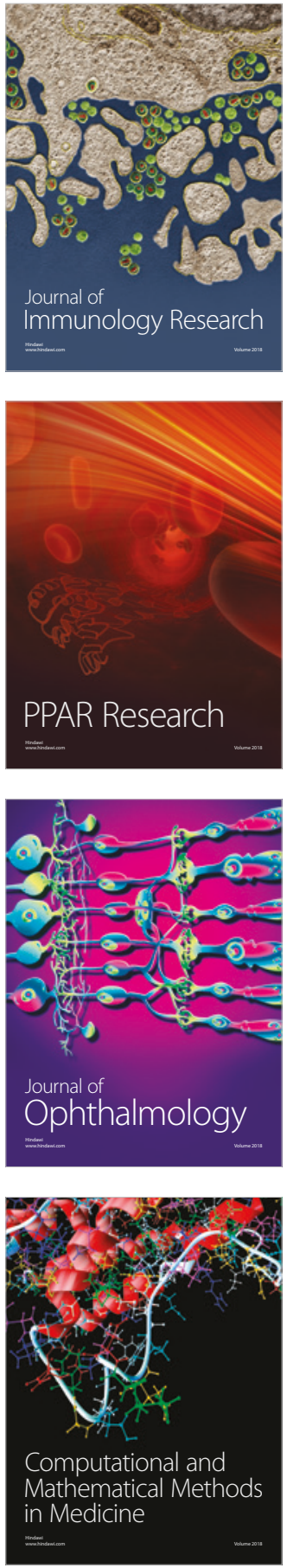

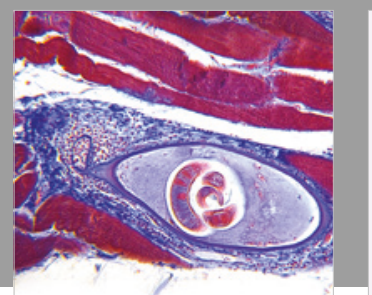

Gastroenterology Research and Practice

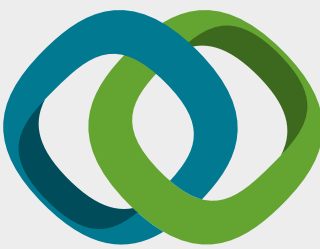

\section{Hindawi}

Submit your manuscripts at

www.hindawi.com
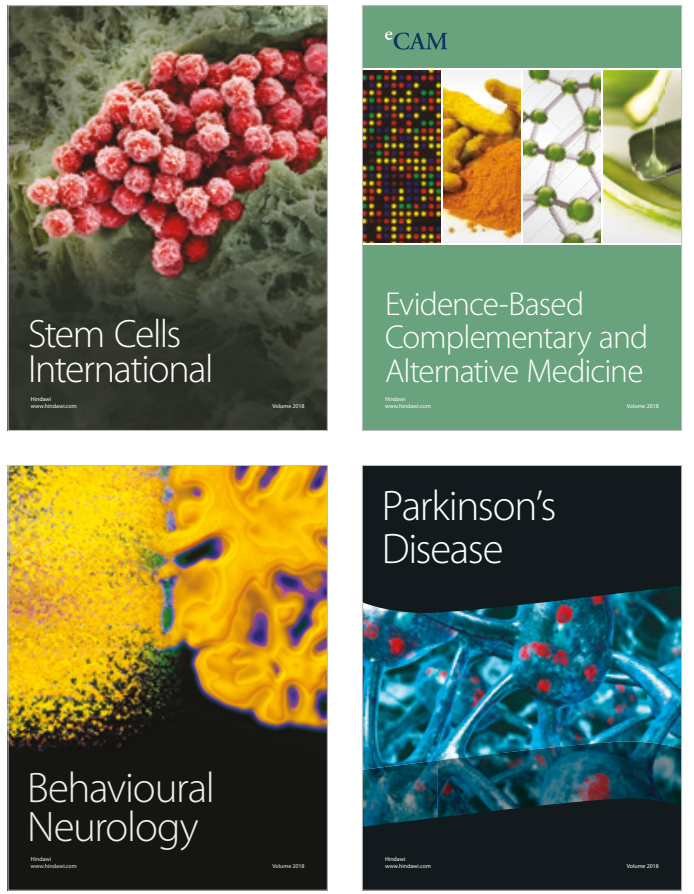

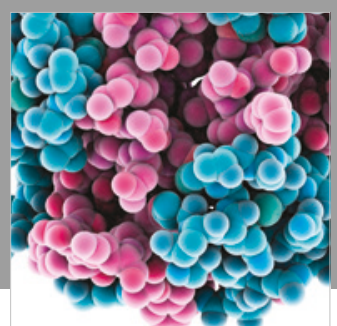

ournal of

Diabetes Research

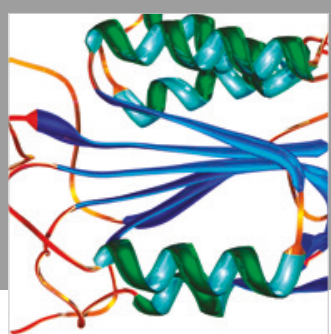

Disease Markers
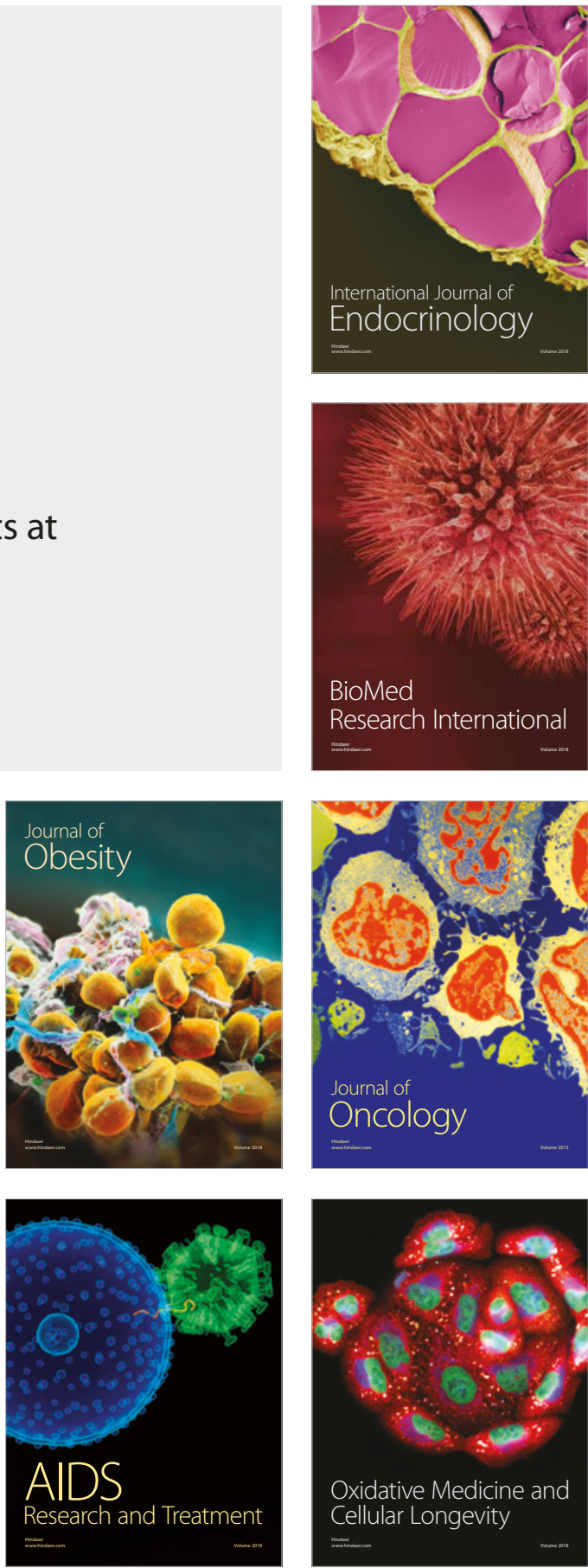